\title{
Treatment can reduce fracture risk after organ transplantation
}

Treatment with bisphosphonates or active metabolites of vitamin D after a solid organ transplantation reduces the risk of fractures during the first year after surgery.

"We and others have shown that solid organ transplantation is followed by rapid bone loss and an increased incidence of fractures, and that initiation of drugs that inhibit bone resorption can prevent the bone loss," explains senior author Elizabeth Shane of Columbia University,

New York. "However, very few studies have been large enough to show that preventing the bone loss also prevents fractures."

The researchers carried out a metaanalysis of the fracture-prevention effect of bisphosphonates or active metabolites of vitamin $\mathrm{D}$ during the first year after solid organ transplantation. Eleven randomized controlled trials involving 780 transplant recipients were included.

Treatment resulted in fewer patients with fractures, fewer fractures overall and fewer vertebral fractures; in addition, BMD increased by $\sim 3 \%$ at the spine and hip.

"These findings provide strong evidence that initiating either bisphosphonates or active metabolites of vitamin D immediately after transplantation improved BMD and reduced fracture risk," summarizes Shane.

The team now plans to quantify bone loss and fractures in patients who receive more current transplant drug regimens to prevent rejection, which include lower doses of prednisone than were used when many of the studies included in the metaanalysis were conducted. They hope to determine whether bone loss and fractures are still as much of a problem as in the past.

\section{Carol Wilson}

Original article Stein, E. M, et al. Prevention of fractures after solid organ transplantation: a meta-analysis. J. Clin. Endocrinol. Metab. doi:10.1210/jc.2011-1448 